\title{
Evaluation of the mechanical properties of plasma-sprayed coating by nanoindentation technology
}

\author{
Wei-Chao Guo ${ }^{1,2}$, Gast Rauchi ${ }^{3}$, Luc Papeleux ${ }^{1}$, Wei-Hong Zhang ${ }^{2}$, \\ François Gitzhofer ${ }^{4}$ And JeAn-Philippe Ponthot ${ }^{1, a}$ \\ 1 LTAS/MN²L, Aerospace \& Mechanical Engineering Department, University of Liège, 4000 Liège, Belgium \\ 2 Engineering Simulation and Aerospace Computing, the Key Laboratory of Contemporary Design \& Integrated \\ Manufacturing Technology, Northwestern Polytechnical University, Xi'an, 710072 Shaanxi, China \\ 3 Department of Advanced Materials and Structures, Centre de Recherche Public Henri Tudor, 4221 Esch-sur-Alzette, \\ Luxembourg \\ 4 Plasma Technology Research Center (CRTP), Department of Chemical Engineering, University of Sherbrooke, \\ Sherbrooke, Québec, Canada J1K 2R1
}

Received 17 February 2011, Accepted 13 June 2012

\begin{abstract}
In this paper, the mechanical properties of plasma-sprayed coatings are evaluated using the technology of nanoindentation. According to the indentation curve (load versus indenter's displacement), which is recorded in an indentation experiment, the mechanical behaviour of the plasma-sprayed coating is investigated via inverse analysis using numerical optimization algorithms. The tip rounding of imperfect indenter is investigated in parameter identification. Subsequently, the hardness on the top surface and on the vertical cross section of the plasma-sprayed coating are investigated. The results show that the hardness on the top surface of the coating depends on the indentation depth. Moreover, the results show that the hardness on the cross section of the coating is significantly affected by the substrate if the indentation impression is too close to the coating-substrate interface.
\end{abstract}

Key words: Nanoindentation / hardness / plasma-sprayed coating / inverse analysis / numerical method

\section{Introduction}

The rapid developments of surface engineering technologies and their successful applications in various sectors of industry have led to increasing demands for assessing the mechanical properties of engineering coatings, whose thickness usually ranges from submicron scales to several hundred microns depending on the application requirements and on the processing conditions. In general, plasma-sprayed coatings as a part of thermal spraying are divided into metallic and non-metallic materials and formed through a group of processes which can deposit the coating materials in a molten or semi-molten state onto a prepared substrate $[1,2]$.

For example, the formation principle of plasmasprayed coatings adopted in [2] is shown in Figure 1. In a plasma jet, the thermal plasma heat source (direct current arc or radio frequency discharge) with temperatures over $8000 \mathrm{~K}$ at atmospheric pressure allows the melting of any material. Powdered materials are injected within the

${ }^{a}$ Corresponding author: JP. Ponthot@ulg.ac.be plasma or plasma jet, where particles are accelerated and molten, or partially molten, see Figure 1a. Before they flatten and solidify onto the substrate, the coating will be built by the layering of splats, see Figure 1b. The thickness of the formed coating normally is in the range of microns or millimetres. At such small scales, the mechanical properties of those coatings, e.g. the toughness, hardness, yield strength, elasticity modulus and the strainstress law cannot be determined by traditional methods, such as compression and tensile testing, since they do not scale well into the micro- and nanoscales. However, at such scales, nanoindentation technology has already been widely employed to characterize the mechanical properties of thin coatings. For relatively thick coatings (e.g. the thickness $>10 \mu \mathrm{m}$ ), conventional indentation technology can provide a simple, efficient and robust means for evaluating the coating's properties [3-8].

In the literature, most of the researchers focused on identification of the hardness on the top surface of coatings $[4-6]$. However, according to the earlier research carried out by Lin [7] and Leigh [8], the hardness of coatings may exhibit some variability in thickness direction 


\section{Nomenclature}

\begin{tabular}{|c|c|}
\hline \multicolumn{2}{|r|}{ Latin characters } \\
\hline$a_{c}$ & Contact area radius in cone $(\mathrm{m})$ \\
\hline$a_{s}$ & Contact area radius in sphere $(\mathrm{m})$ \\
\hline$A_{\text {proj }}$ & Projected contact area $\left(\mathrm{m}^{2}\right)$ \\
\hline$C_{i}$ & Constants \\
\hline$D_{0}$ & Diameter of particle \\
\hline$D_{f}$ & Diameter of splat \\
\hline$D$ & Rate of deformation tensor \\
\hline$D^{e}$ & Elastic part in rate of deformation \\
\hline $\boldsymbol{D}^{p}$ & Inelastic part in rate of deformation \\
\hline$E$ & Young's modulus of investigated material $(\mathrm{Pa})$ \\
\hline$E_{i}$ & Young's modulus of indenter $(\mathrm{Pa})$ \\
\hline$E_{r}$ & Reduced modulus $(\mathrm{Pa})$ \\
\hline$f$ & Yield function $(\mathrm{Pa})$ \\
\hline $\bar{f}$ & Updated yield function (Pa) \\
\hline$F$ & Objective function $\left(\mathrm{m}^{2}\right)$ \\
\hline$h$ & Indentation depth $(\mathrm{m})$ \\
\hline $\bar{h}$ & Experimental indentation depth $(\mathrm{m})$ \\
\hline$h_{c}$ & Contact depth $(\mathrm{m})$ \\
\hline$h_{d}$ & Deviation of penetration depth $(\mathrm{m})$ \\
\hline$h_{\max }$ & Maximum indentation depth (m) \\
\hline$G$ & Shear modulus $(\mathrm{Pa})$ \\
\hline$H$ & Hardness $(\mathrm{Pa})$ \\
\hline $\boldsymbol{H}$ & Hooke stress-strain tensor \\
\hline$K$ & Bulk modulus $(\mathrm{Pa})$ \\
\hline$L x, L y s, L y c$ & Length (m) \\
\hline$m$ & Viscosity exponent \\
\hline$n$ & Work-hardening exponent \\
\hline$N$ & Number of load steps \\
\hline$p$ & Porosity \\
\hline$P$ & Load $(\mathrm{N})$ \\
\hline$P_{\max }$ & Maximum load $(\mathrm{N})$ \\
\hline$Q$ & Material parameter in Voce-type hardening law $(\mathrm{Pa})$ \\
\hline$s$ & Deviator of the stress tensor \\
\hline$S$ & Initial unloading stiffness $\left(\mathrm{N} . \mathrm{m}^{-1}\right)$ \\
\hline$x_{j}$ & Material parameters \\
\hline \multicolumn{2}{|r|}{ Greek characters } \\
\hline$\beta$ & Material parameter in Voce-type hardening law \\
\hline$\delta$ & Kronecker delta \\
\hline $\bar{\varepsilon}_{p}$ & Plastic strain \\
\hline $\bar{\varepsilon}_{v p}^{\bullet}$ & Rate of effective viscoplastic strain \\
\hline$\eta$ & Coefficient of viscosity $\left(\mathrm{Pa} / \mathrm{s}^{-1 / \mathrm{m}}\right)$ \\
\hline$\mu$ & Friction coefficient \\
\hline$\theta$ & Half apex angle of conical indenter $\left(^{\circ}\right)$ \\
\hline$\rho$ & Radius of imperfect indenter tip (m) \\
\hline $\bar{\sigma}$ & Effective stress $(\mathrm{Pa})$ \\
\hline$\sigma_{0}$ & Quasi-static flow stress $(\mathrm{Pa})$ \\
\hline$\sigma_{y}^{0}$ & Initial yield stress $(\mathrm{Pa})$ \\
\hline$\sigma_{v}$ & Current yield stress $(\mathrm{Pa})$ \\
\hline$\sigma_{\text {visco }}$ & Stress due to viscous effect $(\mathrm{Pa})$ \\
\hline$\sigma$ & Cauchy stress tensor \\
\hline$\sigma^{\nabla}$ & Objective rate of Cauchy stress tensor \\
\hline$\tau$ & Tolerance \\
\hline$v$ & Poisson's ratio of investigated material \\
\hline$v_{i}$ & Poisson's ratio of indenter \\
\hline
\end{tabular}


(a)

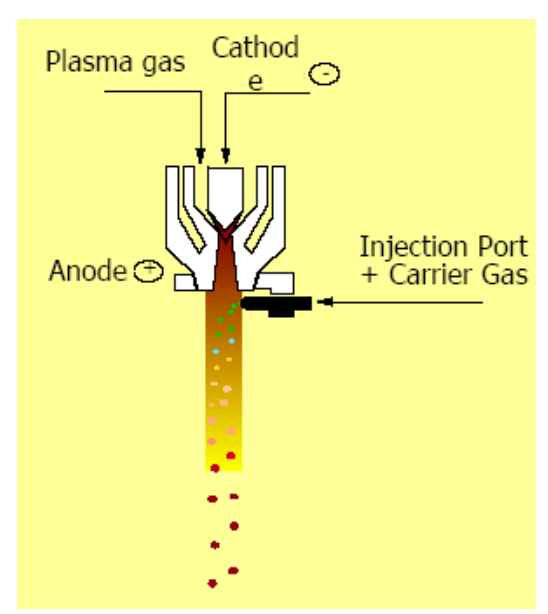

(b)

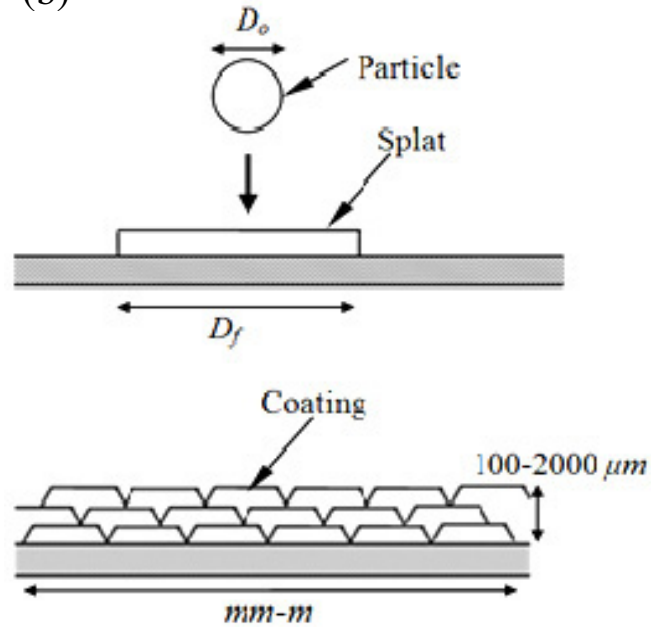

Fig. 1. Principle of plasma-sprayed coating formation $[1,2]$.

due to a heterogeneous microstructure generated during spraying [8] or service conditions producing oxidation or cracking [7]. Therefore, measurement of hardness on the top surface of a coating only is not sufficient enough to explain and describe the mechanical properties, such as the hardness and its relationship with the microstructure of the coating.

In the present paper, the evaluation of a plasmasprayed coating's hardness and the assessment of its relation with the substrate are carried out. First the material parameters of the plasma-sprayed coating are identified via inverse analysis using numerical optimization algorithms based on the experimental data. Then, according to the identified material parameters, the hardness on the top surface and on the vertical cross section of the plasmasprayed coating are investigated.

\section{Theoretical considerations}

\subsection{Theory of hardness measurement}

Usually, for hardness measurement, an indentation instrument is employed. It can continuously register the load $p$ versus the indentation depth $h$, during loading, holding (not necessary) and unloading processes, see Figure 2a. Then, the hardness and Young's modulus can be inferred from the $p-h$ curve by some corresponding estimation methods. In current applications, the most widely used method is proposed by Oliver and Pharr [9], which has been adopted as a standard reference method in ISO-14577 [10],

$$
\begin{gathered}
H=\frac{P}{A_{\text {proj }}} \\
E_{r}=\frac{1}{2} \sqrt{\frac{\pi}{A_{\text {proj }}}} \frac{\mathrm{d} P}{\mathrm{~d} h}
\end{gathered}
$$

$$
\begin{aligned}
h_{c} & =h_{\max }-\frac{2}{\pi}(\pi-2) \frac{P_{\max }}{S} \\
A_{\text {proj }} & =24.5 h_{c}^{2}+\sum_{i=1}^{8} C_{i} h_{c}{ }^{1 / 2^{i-1}}
\end{aligned}
$$

where, $A_{\text {proj }}$ is the projected contact area, see Figure $2 \mathrm{~b}$, which can be calculated by Equation (4). The constants, $C_{i}$ in Equation (4) describe the deviation caused by an imperfect indenter tip. In practice, $C_{i}$ are calibrated by some known materials in experiments, e.g. fused silicon, aluminium $[9,10] . P_{\max }$ is the maximum load and $h_{\max }$ is the maximum indentation depth. $h_{c}$ is the contact depth, see the details in Figure 2b. In the following numerical simulations, $h_{c}$ is obtained from the modelled deformed surface and $C_{i}$ are set as zero if the indenter is designed with a perfect sharp tip. $S=\mathrm{d} P / \mathrm{d} h$ is the initial unloading stiffness, which is calculated at the maximum indentation depth, see Figure 2a. $E_{r}$ is the so-called reduced modulus, which includes the Young's modulus and Poisson's ratio of the indenter $\left(E_{i}, \nu_{i}\right)$ and of the investigated material $(E, \nu)$. The relationship of them is described by

$$
\frac{1}{E_{r}}=\frac{1-\nu^{2}}{E}+\frac{1-\nu_{i}^{2}}{E_{i}}
$$

For a diamond indenter, $E_{i}=1140 \mathrm{GPa}$ and $\nu_{i}=0.07$. Thus, in order to calculate $E$ by Equation (5), $\nu$ should be known. Normally, $\nu$ is set as 0.3 or 0.25 for unknown materials. In this paper, $\nu=0.3$ is assumed for the investigated plasma-sprayed coating.

\subsection{Material model}

Through the nanoindentation experimental measurement, the hardness and reduced Young's modulus of the sample are obtained. Besides this, the further mechanical 
(a)

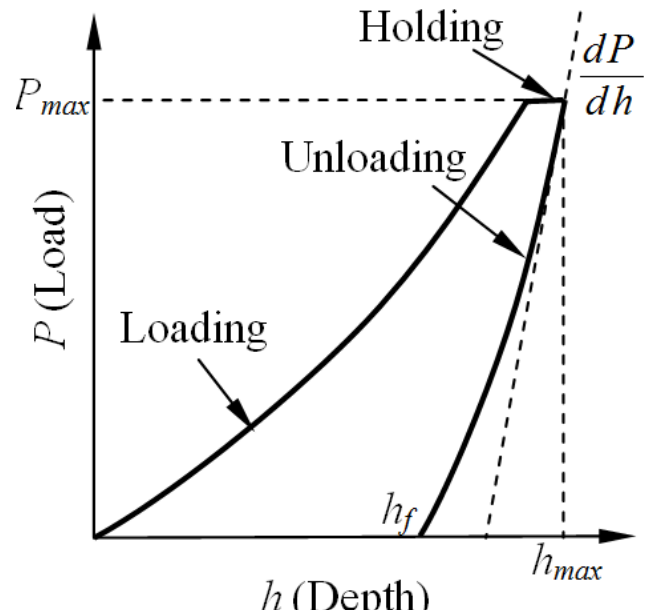

(b)

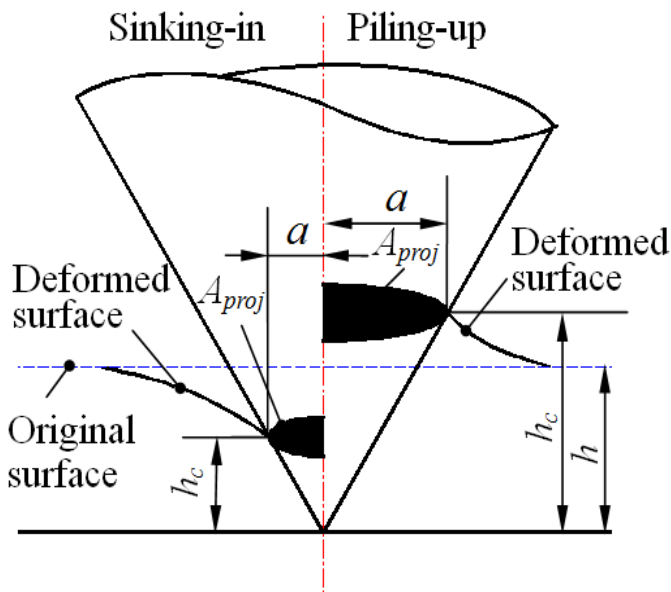

Fig. 2. Schematics of load versus indentation depth curve and indentation profiles with sinking-in and piling-up.

behaviour of the plasma-sprayed coating, e.g. the elastoplastic and viscous behaviour is also interested. In the present paper, the investigated plasma-sprayed coating is made of $\mathrm{Y}_{2} \mathrm{O}_{3}$-stabilized $\mathrm{ZrO}_{2}$. Through the experimental data, see Figure 5, the creep behaviour during the holding process can be seen clearly, which shows that this kind of coating not only has elasto-plastic behaviour, but also exhibits viscous behaviour.

Therefore, according to the mechanical behaviour of the plasma-sprayed coating, a constitutive formulation will be presented for obtaining more mechanical properties the plasma-sprayed coating. The establishment of the formulation in this section considers the work of Ponthot [11], who introduced a unified stress update algorithm for elasto-plastic constitutive equations and then extended it to elasto-viscoplasticity in a finite deformation framework using a corotational formulation, within an updated Lagrangian scheme.

According to [11], the rate of deformation, $\boldsymbol{D}$ can be additively decomposed into an elastic, $\boldsymbol{D}^{e}$ (reversible) and an inelastic, $\boldsymbol{D}^{p}$ (irreversible) part, i.e.

$$
\boldsymbol{D}=\boldsymbol{D}^{e}+\boldsymbol{D}^{p}
$$

The relationship between the rate of strain and the rate of stress is postulated as

$$
\boldsymbol{\sigma}_{i j}^{\nabla}=H_{i j k l}\left(\boldsymbol{D}_{k l}-\boldsymbol{D}_{k l}^{p}\right) \quad \text { or } \quad \boldsymbol{\sigma}^{\nabla}=\boldsymbol{H}:\left(\boldsymbol{D}-\boldsymbol{D}^{p}\right)
$$

where, $\sigma^{\nabla}$ is an objective rate of the Cauchy stress tensor $\boldsymbol{\sigma} . \boldsymbol{H}$ is the Hooke stress-strain tensor (elastic stiffness tensor) which is given by

$$
H_{i j k l}=K \delta_{i j} \delta_{k l}+2 G\left(\delta_{i k} \delta_{j l}-\frac{1}{3} \delta_{i j} \delta_{k l}\right)
$$

where, $\delta$ is the Kronecker delta symbol, $k$ is the bulk modulus and $G$ is the shear modulus of the material.

Plastic deformation is triggered when the stress in the material reaches a given limit. In the material model, the yield function is used to detect an increase of the plastic deformations. It defines a surface which envelops all physically possible stress states in rate-independent plasticity. Stress states inside this contour cause only elastic deformations, while stress states on this yield surface give rise to elastic-plastic deformations. By definition, rateindependent plastic stress states outside the yield contour $f$ are not admissible.

Moreover, the von Mises yield function with $j_{2}$ flow theory for isotropic materials will be chosen as the yield criterion in the following numerical calculations, more details can be seen in $[11,12]$. This yield criterion is frequently assumed for metals and alloys [12]. Furthermore, it offers the numerical advantage that the gradients of the von Mises yield surface, which are used for the numerical solution procedure, are always uniquely defined. Mathematically, the yield function is expressed as,

$$
f\left(\boldsymbol{\sigma}, \sigma_{\nu}\right)=\bar{\sigma}-\sigma_{\nu}=0
$$

where, $\bar{\sigma}$ is the effective stress, i.e. $\bar{\sigma} \sqrt{\frac{3}{2} s: s} ; s$ is the deviator of the stress tensor; $\sigma_{\nu}$ is the current yield stress.

While $f<0$, there is elastic material behaviour. For $f=0$, rate-independent plastic material behaviour takes place. Herein, plastic hardening with an associative flow rule is considered, including non-linear isotropic hardening. The evolution of yield stress, $\sigma_{\nu}$ can be closely approximated by the Voce-type hardening law,

$$
\sigma_{\nu}=\sigma_{y}^{0}+\varrho\left[1-\exp \left(-\beta \varepsilon_{p}\right)\right]
$$

where, $\sigma_{y}^{0}$ is the initial yield stress. $Q$ and $\beta$ are nonlinear isotropic hardening parameters. In addition, if the materials are considered with viscosity, e.g. if the viscosity is described as the Cowper-Symonds law,

$$
\sigma_{\text {visco }}=\sigma_{0}\left(\frac{\bar{\varepsilon}_{v p}^{\bullet}}{\eta}\right)^{\frac{1}{m}}
$$




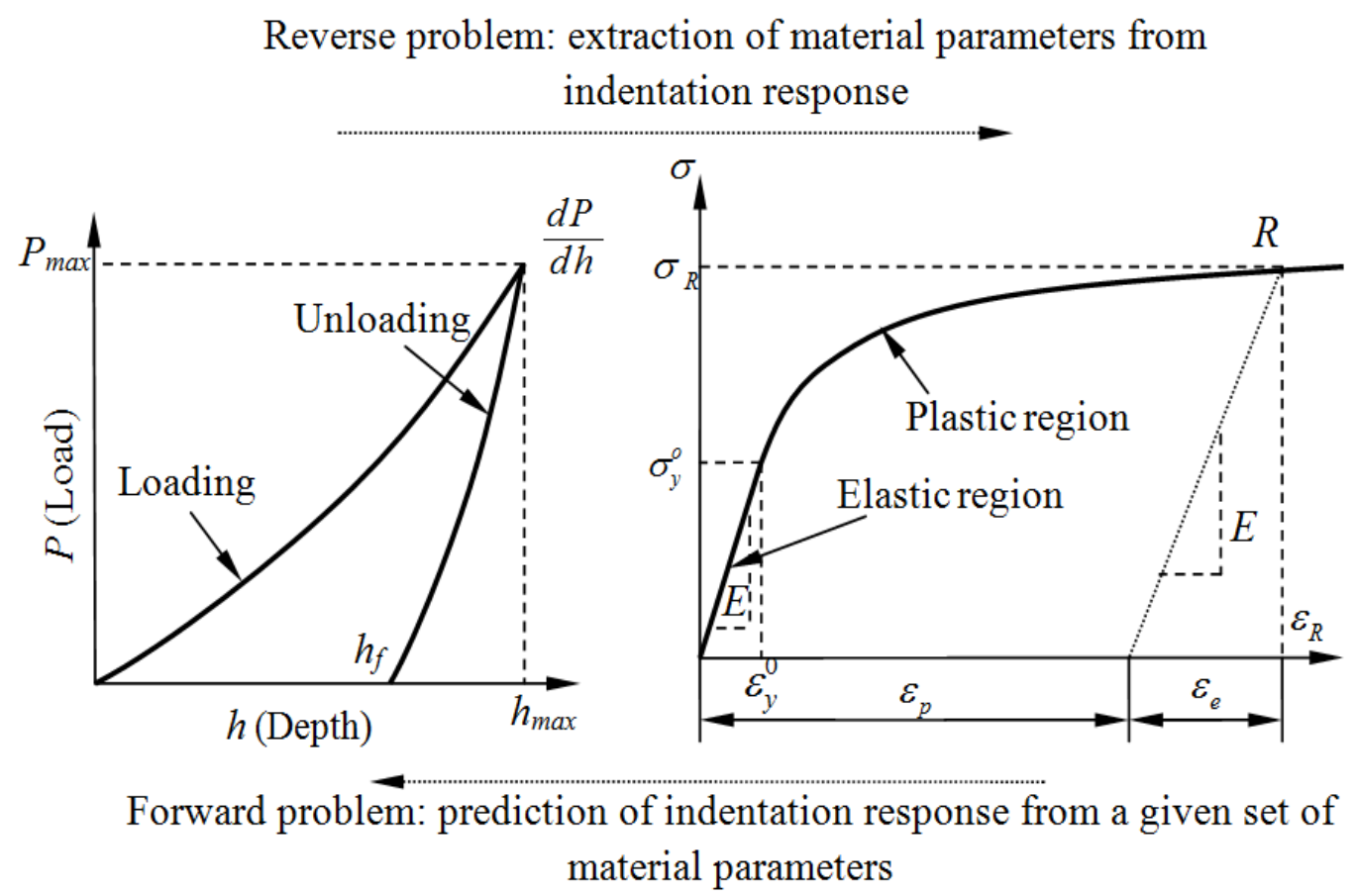

Fig. 3. Graphic depiction of the forward and inverse problems in indentation for elasto-plastic materials [20].

where, $\sigma_{0}$ is the quasi-static flow stress. $\bar{\varepsilon}_{v p}^{\bullet}$ is the rate of effective viscoplastic strain. $\eta$ is a constant and $m$ is the viscosity exponent. A new constraint is defined in the viscoplastic range according to $[11,13]$,

$$
\bar{f}=\bar{\sigma}-\sigma_{\nu}-\sigma_{\text {visco }}=0
$$

In the elastic regime, both $f$ and $\bar{f}$ are equivalent because in this case, $\bar{\varepsilon}_{v p}^{\bullet}=0$ and $\bar{\sigma} \leq \sigma_{\nu}$. So that one has $\bar{f} \leq 0$.

\section{Methods for parameter identification}

\subsection{Forward and inverse analyses}

The problems solved by indentation testing are generally divided into two kinds. One is the so-called "forward problem" [14], another is the "inverse problem" [15]. In the forward algorithm, a unique indentation response (e.g. $P-h$ curve) from a given set of material properties (e.g. $E, \sigma_{y}^{0}$ and $\nu$ for elasto-plastic materials) $[16,17]$ can be calculated, whereas the inverse algorithm enables the extraction of material properties from a given set of indentation data $[15,18-20]$. The relationship between these two algorithms can be seen in Figure 3.

In the literature, many researchers focused on using the forward analysis to calculate the hardness [9,19], and using the inverse indentation method to evaluate the relation of strain-stress [21] and analyze the elasto-plastic and elasto-viscoplastic behaviour [22-25] etc. In the following parts, first, an algorithm is presented to identify the material parameters of the investigated plasma-sprayed coating through inverse analysis. Subsequently, the hardness on the top surface and on the cross section of the plasma-sprayed coating are investigated utilizing forward analysis based on the identified material parameters.

\subsection{Optimization algorithm for parameter identification}

In order to identify the material parameters from the $p-h$ curve registered during the loading and unloading processes in an experiment, the inverse analysis mentioned in the foregoing part and based on numerical optimization has to be performed. The key idea of this procedure is to model the indentation test with a numerical method, i.e. the finite-element method, using a starting set of material parameters and quantifying the difference between the modelled indentation curve and the experimental curve through an objective function. Subsequently, the material parameters used in the finite element model are updated using some algorithms from numerical optimization in order to minimize the objective function. When they lead to a good agreement between the experimental and numerical results, the identified material parameters can be used as equivalent values for the investigated material.

Herein, the objective function to be minimized by numerical optimization algorithm is made up of the difference between the experimental and modelled displacement-into-surface, $\bar{h}$ and $h$, respectively, which are the function of the independent load, $P$.

$$
F=\frac{1}{2 N} \sum_{i=1}^{N}\left[h\left(p_{i}\right)-\bar{h}\left(P_{i}\right)\right]^{2}
$$




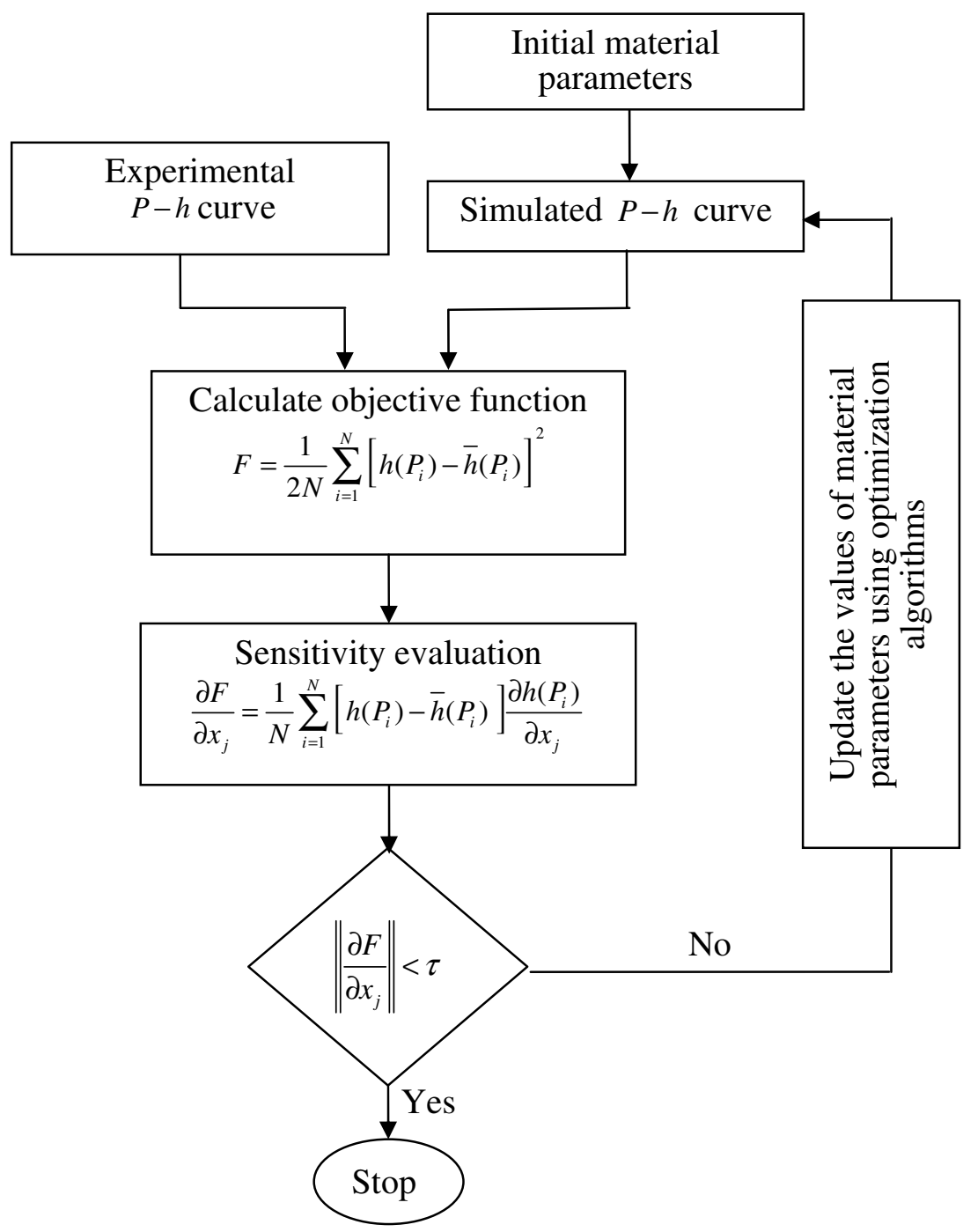

Fig. 4. The process of optimization.

Therefore, the sensitivity analysis is defined as the derivative of the objective function with respect to these material parameters.

$$
\frac{\partial F}{\partial x_{j}}=\frac{1}{N} \sum_{i=1}^{N}\left[h\left(P_{i}\right)-\bar{h}\left(P_{i}\right)\right] \frac{\partial h\left(P_{i}\right)}{\partial x_{j}}
$$

where, $x_{j}$ represents the material parameters. After defining the objective function and sensitivities, the objective function can be minimized using some gradient-based numerical optimization algorithms. In the present research, the cascade optimization methodology which is a combination of Levenberg-Marquardt and conjugate gradient algorithms is adopted. This optimization methodology is proposed by Ponthot, its efficiency and robustness are compared with other optimization algorithms seen in( $[26]$. When $F$ drops below a predefined tolerance, the optimization process will be stopped and optimized material parameters will be written down. The whole procedure is shown in Figure 4.

\section{Results and discussion}

\subsection{Experimental results}

In the real indentation experiment, a sample with $\sim 100 \mu \mathrm{m}$ thickness of plasma-sprayed coating $\left(\mathrm{ZrO}_{2}-\right.$ $\mathrm{Y}_{2} \mathrm{O}_{3}$ ) formed on a stainless steel substrate was measured. The proportion of $\mathrm{ZrO}_{2}$ and $\mathrm{Y}_{2} \mathrm{O}_{3}$ is 93:7 (mol) and the porosity of the coating is in the range of $5-10 \%$. In this experiment, the nano-triboindenter instrument [27] equipped with a Berkovich indenter was used. The measurement procedure involves continuous monitoring of the load and of the indenter's displacement during the loading, holding and unloading processes; see left figure in Figure 5, where the experimental data are represented by squares. The indentation processes are executed by force control and the maximum load equals $4908 \mu \mathrm{N}$. The loading history can be seen in the right figure in Figure 5. From 0 to $5 \mathrm{~s}$, the loading process takes place. Then the loading is held at maximum load for $2 \mathrm{~s}$. After that, the unloading process is performed from 7 to $12 \mathrm{~s}$. 

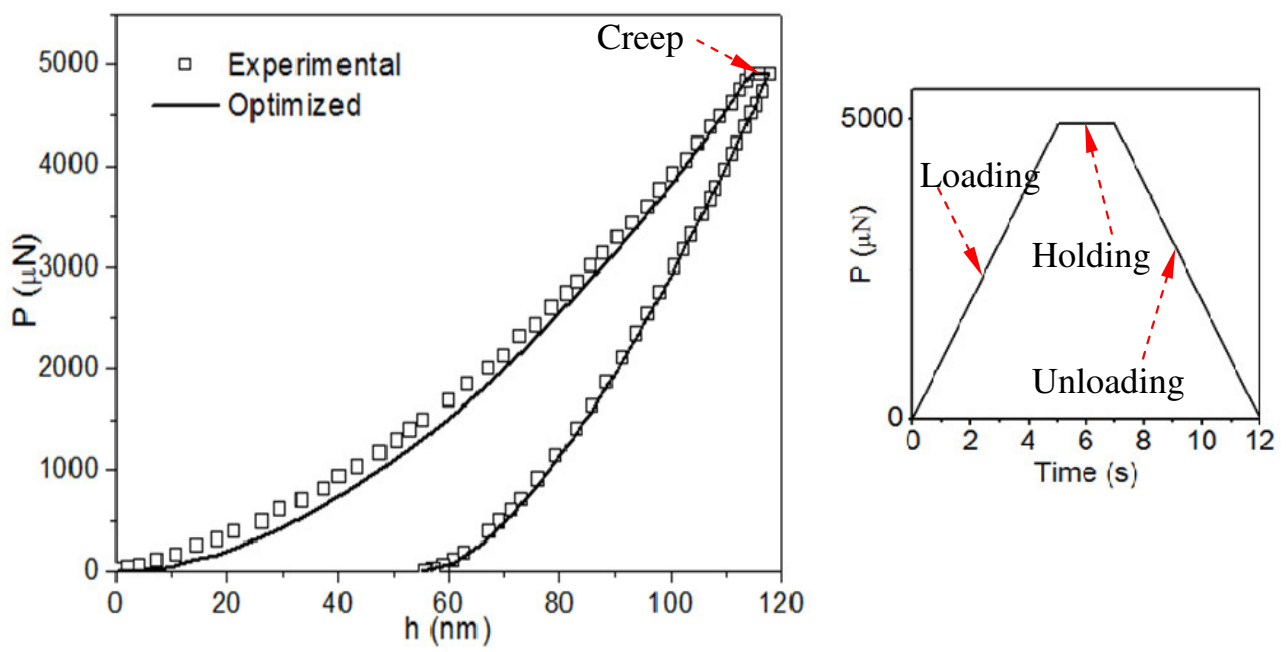

Fig. 5. Optimized $p-h$ curves obtained by perfect conical indenter compared to the experimental data.

Correspondingly, the measured hardness, $H$ equals 16.5 GPa, and reduced Young's modulus, $E_{r}$ equals $192 \mathrm{GPa}$. If the Poisson's ratio of the plasma-sprayed coating is assumed as 0.3, its Young's modulus, $E=$ 209.9 GPa, which is calculated according to Equation (5).

\subsection{Identification of the material parameters of the plasma-sprayed coating}

According to the experimental data, the material parameters of the plasma-sprayed coating can be evaluated by inverse analysis using numerical optimization. In the following parts, all the simulation studies are performed in the finite-element code METAFOR, which performs powerfully in computations of nonlinear materials and large deformations [28].

Because the coating's thickness of the measured sample is $\sim 100 \mu \mathrm{m}$, the maximum indentation depth is lower than $120 \mathrm{~nm}$, see Figure 5. The indentation depth is below $1 / 800$ of the coating's thickness. Therefore, it can be said that the experimental $P-h$ curve is not affected by the substrate as the researchers demonstrated that for hard coating and soft substrate material, normally, the measured results are no longer affected by the substrate when the indentation depth is under $1 / 30$ of the thickness of coating [29]. Thus, herein, in order to simplify the problem and save the computer time, $2 \mathrm{D}$ finite-element model is adopted and it is modelled only with the coating material, see Figure 6. The 2D axisymmetric finiteelement is defined with $7 \mu \mathrm{m}$ radius and $7 \mu \mathrm{m}$ height. It is established using 1062 four-node quadrilateral elements, where a finer mesh near the contact region and a gradually coarser mesh further from the contact region are designed to ensure numerical accuracy. Moreover, the horizontal and vertical displacements are fixed at central and bottom lines as boundary conditions. In the experiment, the used indenter is a three-sided Berkovich indenter. Therefore, as proposed by Albrecht [21] for 2D computations, the Berkovich indenter can be modelled by a conical indenter with the half apex angle of $\theta=70.3^{\circ}$. Furthermore, the Coulomb's friction model is assumed in the contact region with the friction coefficient, $\mu=1.5$. The loading history, exactly corresponds to the one performed in the experiment, which can be seen in the right figure in Figure 5.

The optimized $p-h$ curve compared to the experimental data is shown in Figure 5a. It is clearly seen that the optimized $p-h$ curve has a good agreement with the experimental one. The final identified coating's material parameters are listed in the first rows of Table 1 . The simulated hardness equals $15.2 \mathrm{GPa}$. The difference is $7.9 \%$ compared to the experimental hardness, $H=16.5 \mathrm{GPa}$. Moreover, the identified Young's modulus is compared to the experimental one, $E=209.9 \mathrm{GPa}$, the difference is $2.9 \%$.

However, we can see in Figure 5 that, when the indenter starts penetrating into the specimen, the optimized loading curve is lower than the experimental curve. This is supposed to be due to the tip rounding of the indenter used in the experiment because every indenter never has a perfect sharp tip as designed in practical application [3133]. For this reason, a conical indenter with a rounded tip is modelled like the one shown in Figure 7 . The radius of the indenter rounded tip, $\rho$ is defined as a variable parameter in the optimization procedure. According to the geometry of this imperfect conical indenter, the contact radius, $a_{c}$ can be written as

$$
a_{c}=\left(h_{c}+h_{d}\right) \tan \theta
$$

where, $h_{d}$ is the deviation of penetration depth for the imperfect indenter. It is expressed by

$$
h_{d}=\rho\left(\sin ^{-1} \theta-1\right)
$$

Moreover, the contact radius $a_{s}$ which is produced by the rounded tip can be calculated as

$$
a_{s}=\rho \cos \theta
$$




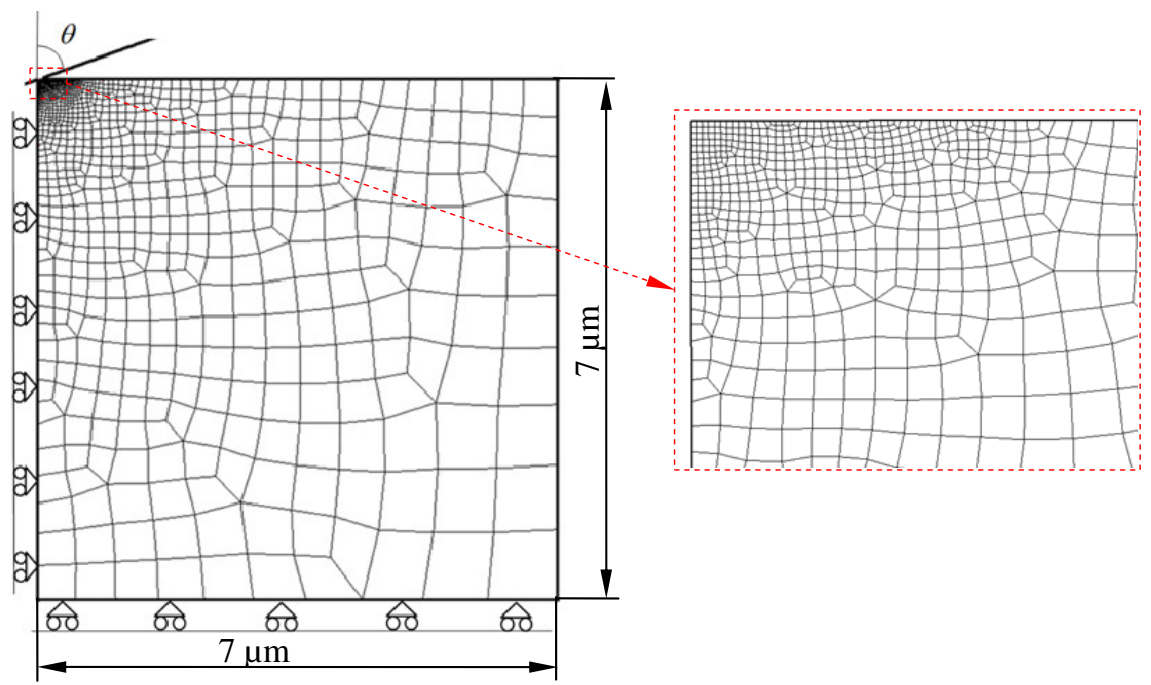

Fig. 6. 2D axisymmetric finite-element model for parameter identification.

Table 1. The identified material parameters for the investigated plasma-sprayed coating.

\begin{tabular}{cccccccc}
\hline$\rho(\mathrm{nm})$ & $E(\mathrm{GPa})$ & $\sigma_{y}^{0}(\mathrm{GPa})$ & $Q(\mathrm{GPa})$ & $\beta(\mathrm{GPa})$ & $\eta\left(\mathrm{GPa} / \mathrm{s}^{1 / \mathrm{m}}\right)$ & $m$ & $H(\mathrm{GPa})$ \\
\hline 0 & 203.8 & 8.99 & 15.89 & 5.41 & 0.046 & 0.54 & 15.2 \\
\hline 219 & 167.6 & 7.70 & 0.03 & 8.34 & 0.056 & 2.06 & 11.5 \\
\hline
\end{tabular}

The optimized load-displacement curve compared to the experimental data is shown in Figure 8. It is clear that the optimized load-displacement curve has a good agreement with the experimental curve. The corresponding identified material parameters for this coating and the optimized radius of the rounded tip are listed in Table 1. As can be seen in the second row of this table, the optimized radius of the rounded tip equals $219 \mathrm{~nm}$. It is in the reasonable range according to the investigation [10]. However, it is noted that at the maximum penetration depth $h_{\max }=118 \mathrm{~nm}$, the contact depth $h_{c}=114 \mathrm{~nm}$ is directly measured from the finite-element model. Thus the ratio of $a_{s} / a_{c} \approx 0.21$ can be calculated according to Equations (15) and (17). This means that the conical indenter used in the experiment is worn out very much and about $21 \%$ part of the contact area is a spherical contact area.

In order to calculate the projected contact area produced by the imperfect indenter, the constants $C_{i}$ in Equation (4) have to be calibrated. Here, only $C_{i}(i=$ $1,2,3,4)$ are calibrated because the latter terms in Equation (4) affect $A_{\text {proj }}$ less. The identified material parameters listed in the second rows of Table 1 are used to calibrate $C_{i}$. As a consequence, $A_{\text {proj }}$ for this imperfect indenter is written as

$$
\begin{array}{r}
A_{\text {proj }}=24.5 h_{c}^{2}+677.5 h_{c}-385.1 h_{c}^{1 / 2}+157.7 h_{c}^{1 / 4} \\
+3891.5 h_{c}^{1 / 8}
\end{array}
$$

The corresponding calculated hardness and Young's modulus can be seen in the second rows of Table 1. The differences of the hardness and Young's modulus are over than $30 \%$ and $18 \%$, respectively, in comparison with the

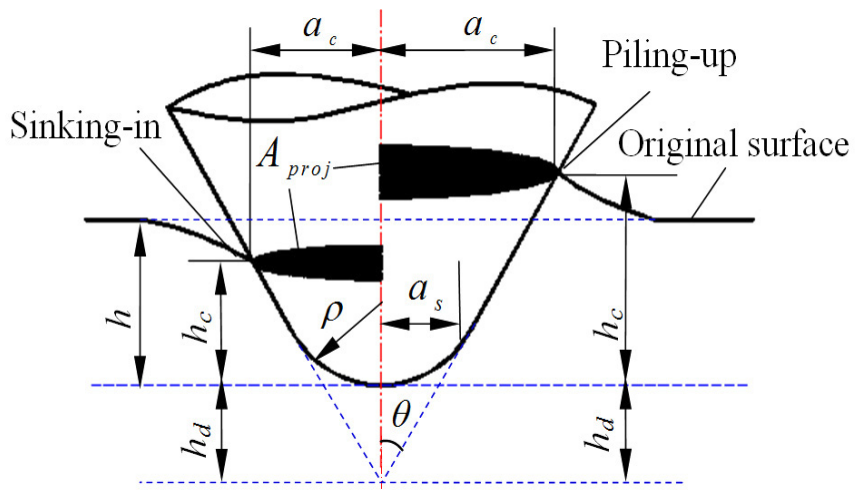

Fig. 7. Schematic of the indentation profiles for an imperfect conical indenter with the sinking-in and the piling-up [30].

experimental ones. Concerning to the large differences between simulated data and experimental data, they may be led by the calculation of $A_{\text {proj. }}$. In the experiment $A_{\text {proj }}$ is calculated using Equation (4). However, the constants $C_{i}$ in Equation (4) may be calibrated when the indenter was new. After the indenter was worn, the constants $C_{i}$ no longer were exact for this worn indenter. That is due to the fact that in the experiment, the calculated $A_{\text {proj }}$ equals $3.0 \times 10^{5} \mathrm{~nm}^{2}$. However, according to Equation (18), for the imperfect indenter with the tip radius $\rho=219 \mathrm{~nm}$, at the same penetration depth, $A_{\text {proj }}$ equals $4.03 \times 10^{5} \mathrm{~nm}^{2}$. Thus the calculated hardness for identified material parameters is smaller than the hardness obtained in the experiment. 


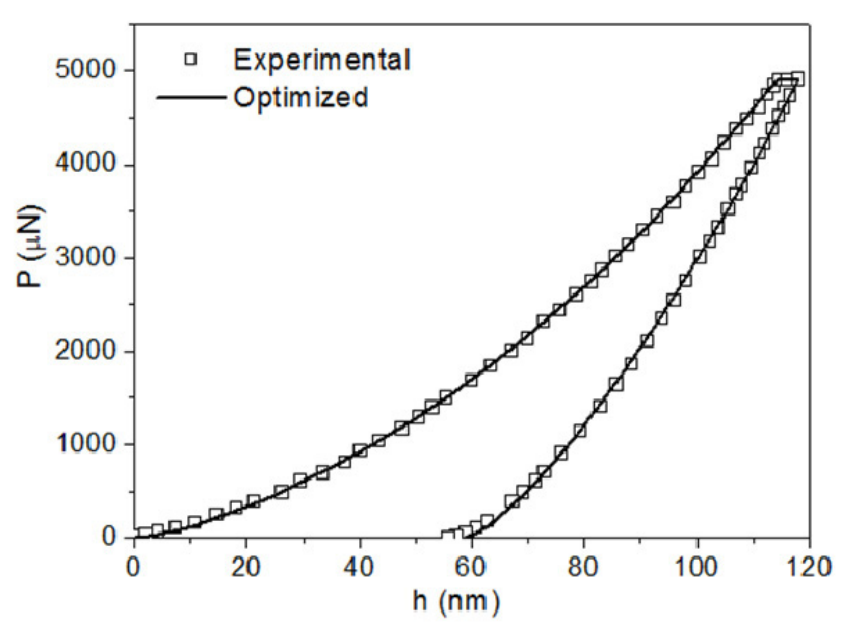

Fig. 8. Optimized $p-h$ curves obtained by imperfect conical indenter compared to the experimental data.

Furthermore, when plasma-sprayed coating is being generated, porosity exists in the coating. According to the investigations $[34,35]$, the Young's modulus of a coating is dependent on this porosity. The Young's modulus will decrease significantly with an increase of the porosity. The relationship of them can be fitted according to experimental results for a $\mathrm{ZrO}_{2}-\mathrm{Y}_{2} \mathrm{O}_{3}$ coating [34],

$$
E=E_{0} \exp \left(a_{e} p\right)
$$

with $E_{0}=221 \pm 4 \mathrm{GPa}$ and $a_{e}=2.7 \pm 0.5$, where $p$ is the porosity. Thus, for a coating with $10 \%$ porosity, its Young's modulus would decrease to 169 GPa. Simultaneously, it is reminded that the Young's modulus equals 167.6 GPa obtained by the imperfect conical indenter in the previous part. They have a good agreement. This denotes that the experimental Young's modulus may have a large error.

Furthermore, it is clear that the optimized loaddisplacement curve which takes into account the tip rounding is close to the experimental one. Therefore, it is believed that the identified material parameters determined by the imperfect conical indenters are more reliable because the corresponding load-displacement curve is almost identical compared to the experimental curve. As a consequence, the material properties of the plasmasprayed coating can be presented by the material parameters which are evaluated by the imperfect conical indenter. Thus in the following hardness investigations, the mechanical behaviour of the coating is described by the identified material parameters in the second row of Table 1.

\section{Hardness testing}

\subsection{Hardness on top surface of coating}

The hardness on the top surface of coating is focused on in many of the published papers [6-8]. It represents the

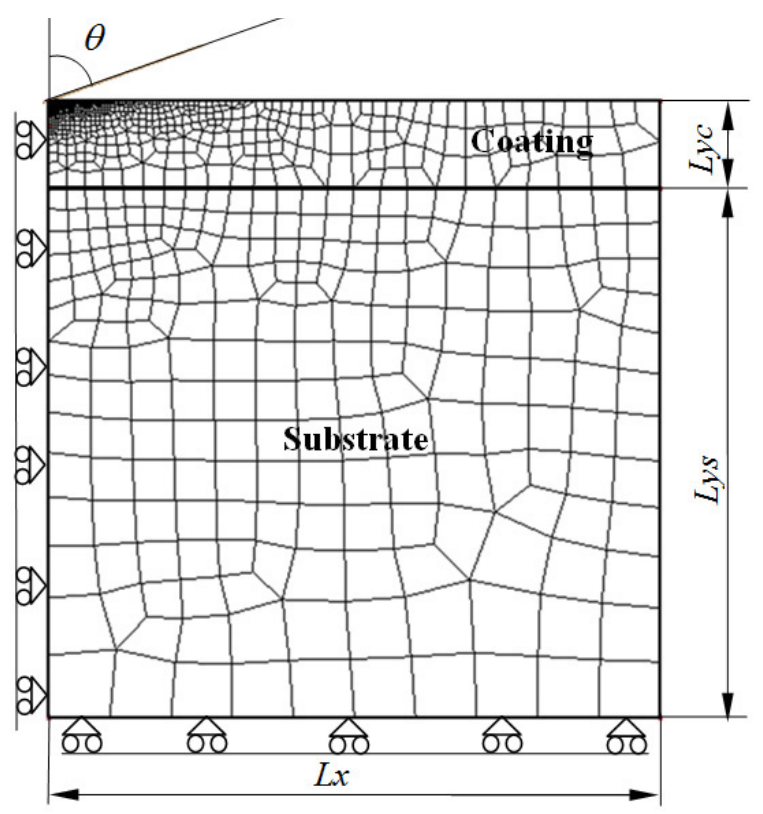

Fig. 9. 2D axisymmetric finite-element model for coating material.

work condition of coatings in practical applications. However, it is reported that hardness depends on the indentation depth. In order to investigate this problem, another $2 \mathrm{D}$ axisymmetric finite-element model with a perfect conical indenter is modelled using 958 four-noded quadrilateral elements. There are 737 elements for the coating and 221 elements for the substrate. A finer mesh near the contact region and a gradually coarser mesh further from the contact region are designed to ensure numerical accuracy, see Figure 9. The model is designed with the radius, $L x=7 \mu \mathrm{m}$. The thickness of the coating is presumed as $L y c=1 \mu \mathrm{m}$ and the thickness of the substrate is presumed as Lys $=6 \mu \mathrm{m}$. Herein, the substrate is stainless steel, its plastic behaviour is approximated by the power law description according to Mata [19],

$$
\sigma_{\nu}=\sigma_{y}^{0}\left(\frac{E}{\sigma_{y}^{0}} \bar{\varepsilon}_{p}\right)^{n}
$$

The work-hardening exponent, $n=0.19$, the Young's modulus, $E=200 \mathrm{GPa}$, the initial yield stress, $\sigma_{y}^{0}=$ $675 \mathrm{MPa}$ and the Poisson's ratio is set as 0.3 .

Because a perfect sharp conical indenter is used in this part, the projected contact area will be calculated by Equation (4) and the constants $C_{i}$ are set to zero. The hardness is identified at the maximum load, $P_{\max }$ varies from 100 to $13195 \mu \mathrm{N}$, respectively. The hardness versus the ratio of the indentation depth and the coating's thickness is shown in Figure 10. It can be seen, at the range of ultimate low loads, i.e. the indentation depth is lower than about $7.5 \%$ of the coating's thickness, the hardness is nearly constant and agrees well with the calculated hardness, which is already obtained by the pure coating model shown in Figure 6. However, the hardness decreases significantly with an increase of $h_{\max }$ as expected, because when $h_{\max }$ increases more, the identified hardness of the 


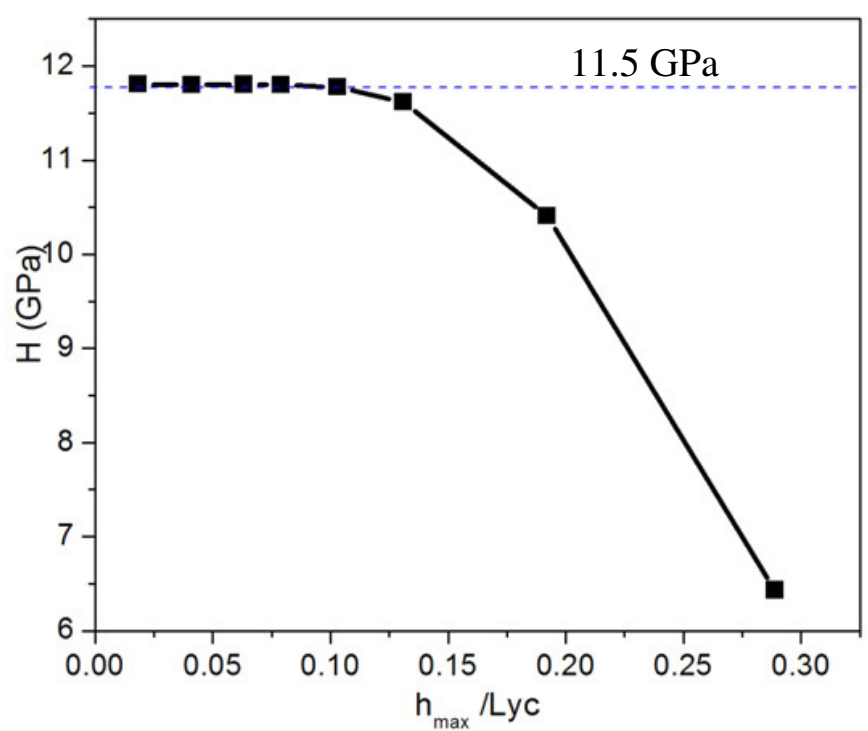

Fig. 10. Hardness versus the ratio of the indentation depth versus the coating's thickness (blue dash line represents the hardness of $11.5 \mathrm{GPa}$. It is obtained by the pure coating model shown in Fig. 6).

coating is influenced more by the substrate. Therefore, as usual, the indentation depth is required that it should not exceed $10 \%$ of the thickness of coating [36] for eliminating the substrate effects. Whereas, on the other hand, an indentation depth as large as possible should be used in order to eliminate indentation size effect (ISE) and specimen roughness effect which are usually more significant at lower indentation depth. Thus, the definition of the critical ratio of coating thickness to indentation depth is a challenge in identification of thin coatings. Anyway, the ISE and specimen roughness effect are beyond the aims of this paper.

\subsection{Hardness on vertical cross section}

In [6-8], researchers measured the hardness on the top surface of a coating, as stated in the previous section. The hardness is normally reported as an average value of a certain number of readings from random indentations on the top surface of the coating. However, such hardness is not sufficient to understand the mechanical properties. It is necessary to evaluate the hardness within coating and assess its relation with the substrate. Herein, a 3D model is designed and shown in Figure 11 to describe the schematic of different indentation impressions and micro-structural characteristics of the finite-element models, which are used in the following investigations.

Herein, a perfect conical indenter with the half apex angle of $\theta=70.3^{\circ}$ is used. In order to obtain the same projected contact areas, in the following computations, the load is executed through control of the displacement of the indenter. The maximum indentation depth is set to $h_{\max }=118 \mathrm{~nm}$, which is nearly same as the depth of the indenter reached in the experiment. As a consequence,

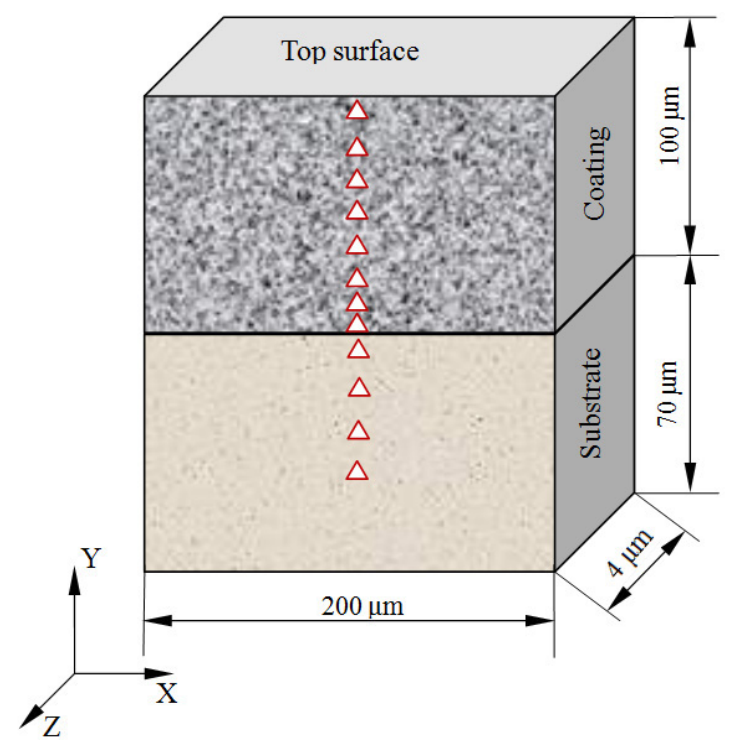

Fig. 11. Schematic of different indentation impressions and microstructural characteristics of the used model.
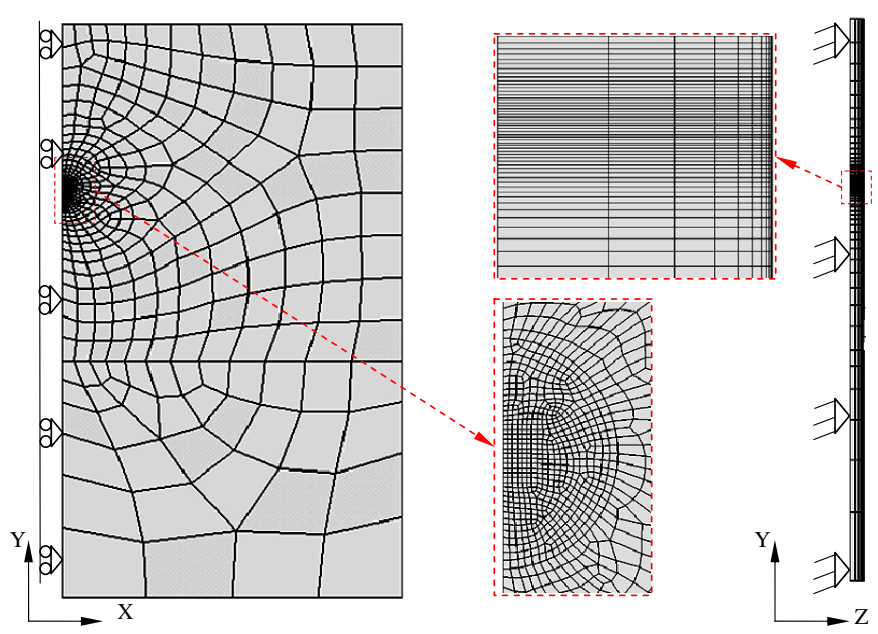

Fig. 12. 3D finite-element model for hardness tests. The indentation position is on the distance of $50 \mu \mathrm{m}$ from the coating-substrate interface.

the radius of projected contact area at the maximum indentation depth is about $329 \mathrm{~nm}$ evaluated according to Equation (4). Thus, for decreasing the number of elements and saving computer time, the size of the used model is designed with $200 \mu \mathrm{m}$ length and $4 \mu \mathrm{m}$ height. The thickness of the coating is $100 \mu \mathrm{m}$ and the thickness of the substrate is assumed as $70 \mu \mathrm{m}$, see Figure 11.

On the cross section of the coating, seven hardness values are calculated, which are positioned at a distance of $0.52,1.25,2.5,25,50,75,97.5$ and $98.96 \mu \mathrm{m}$ from the coating-substrate interface, respectively. Besides this, on the cross section of the substrate, the hardness positioned at $0.52,5,10$ and $40 \mu \mathrm{m}$ from the coating-substrate interface are calculated. Therefore, a total of twelve 3D finiteelement models is designed for the foregoing hardness investigations. The half symmetric 3D finite-element 


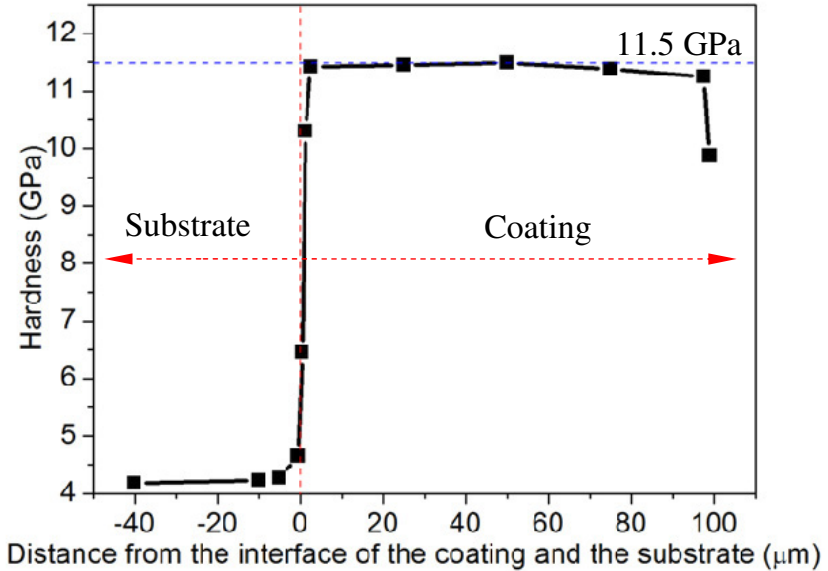

Fig. 13. Hardness distributions on the cross section of the sample (blue dash line represents the hardness of $11.5 \mathrm{GPa}$. It is obtained by the pure coating model shown in Fig. 6).

model for the hardness testing on the distances of $50 \mu \mathrm{m}$ from the coating-substrate interface within coating is shown in Figure 12. This model is established by 11870 eight-noded 3D solid elements. There are 11510 elements in the coating area and 360 elements for the substrate. A finer mesh is designed near the contact region to ensure the calculation accuracy. At maximum load, more than 800 contact nodes are active in the contact region. The bottom surface of the model located on the $X-Y$ plane is fixed as boundary condition and the surface on $Y-Z$ plane is defined as symmetric surface.

The hardness distributions on the cross section of the sample are shown in Figure 13. It can be seen, within the coating, where the indentation positions are far from the coating-substrate interface and the top surface, the hardness is less affected by boundary effects. The maximum hardness is $11.5 \mathrm{GPa}$, which appears in the middle of the coating. It is equivalent to the calculated hardness, $H=11.5 \mathrm{GPa}$ which is obtained by the pure coating model shown in Figure 6.

However, it must be noted that the close to the interface, coating's hardness is affected significantly by the substrate. It can be seen that the hardness of the coating decreases when the indentation impression is close to the coating-substrate interface, because the substrate is much softer than the coating. Particularly, the hardness at a distance of $0.52 \mu \mathrm{m}$ from the coating-substrate interface within the coating decreases to $6.5 \mathrm{GPa}$ as the substrate is less stiff than the coating. Besides this, when the indentation position is too close to the top surface of the coating, the hardness decreases again because the top surface is not constrained.

\section{Conclusions}

In the present work, the mechanical properties of a plasma-sprayed coating are evaluated using inverse analysis based on nanoindentation testing. The tip rounding of an imperfect indenter is investigated in the parameter identification. First, the material parameters of the plasma-sprayed coating are determined by a conical indenter with a perfect sharp tip. The optimized $p-h$ curve has a good agreement with the experimental data. Second, the tip rounding of imperfect conical indenter is considered because the optimized $p-h$ curve is lower than the experimental data at the beginning of loading curve. The result shows that the optimized $p-h$ curve, which includes the effect of tip rounding, is close to the experimental data. Subsequently, based on the identified material parameters, the hardness on the top surface of the plasma-sprayed coating as a function of the indentation depths is studied. The results show that the measured hardness will decrease significantly with an increase of the indentation depth. Furthermore, the relation of the hardness on the vertical cross section of the coating and the substrate is assessed. The results show that the hardness on the cross section of the coating will decrease largely under the effect of the soft substrate, if the indentation impression is close to the coating-substrate interface.

Acknowledgements. The support of W.C. Guo and J.P. Ponthot by the "Direction Générale des Relations Extérieures de la région Wallonne", through the grant "Commission Mixte Québec-Wallonie-Bruxelles" is gratefully acknowledged. The authors also thankfully acknowledge the financial support of the Fonds National de la Recherche of the Grand Duchy of Luxembourg, under the grant number TR-PHD BFR 06/027. The authors thank Prof. F. Gitzhofer and Mr. L. Jia of the Plasma Technology Research Centre of University of Sherbrooke in Canada for providing the experimental indentation data on the plasma-sprayed coating.

\section{References}

[1] P. Fauchais, Understanding plasma spraying, J. Phys. D: Appl. Phys. 37 (2004) 86-108

[2] F. Gitzhofer, Induction plasma technology applied to the combinatorial synthesis of coatings using suspensions and solutions precursors, The 3th international workshop RIPT (Les Rencontres Internationales sur la Projection Thermique) University of Sciences and Technologies of Lille, France, 2007

[3] Z. Mohammadi, A.A. Ziaei-Moayyed, A.S.M. Mesgar, Adhesive and cohesive properties by indentation method of plasma-sprayed hydroxyapatite coatings, Appl. Surf. Sci. 253 (2007) 4960-4965

[4] Z.J. Yin, S.Y. Tao, X.M. Zhou, C.X. Ding, Evaluating microhardness of plasma sprayed $\mathrm{Al} 2 \mathrm{O} 3$ coatings using Vickers indentation technique, J. Phys. D - Appl. Phys. 40 (2007) 7090-7096

[5] K.D. Bouzakis, A. Lontos, N. Michailidis, O. Knotek, E. Lugscheider, K. Bobzin, A. Etzkorn, Determination of mechanical properties of electron beam-physical vapor deposition-thermal barrier coatings (EB-PVD-TBCs) by means of nanoindentation and impact testing, Surf. Coatings Technol. 163-164 (2003) 75-80

[6] J.F. Li, X.Y. Wang, H. Liao, C.X. Ding, C. Coddet, Indentation analysis of plasma-sprayed $\mathrm{Cr} 3 \mathrm{C} 2-\mathrm{NiCr}$ coatings, J. Mater. Sci. 39 (2004) 7111-7114 
[7] C.K. Lin, C.C. Berndt, Statistical analysis of microhardness variations in thermal spray coatings, J. Mater. Sci. 30 (1995) 111-117

[8] S.H. Leigh, C.C. Berndt, Evaluation of off-angle thermal spray, Surf. \& Coatings Technol. 89 (1997) 213-224

[9] W.C. Oliver, G.M. Pharr, An improved technique for determining hardness and elastic modulus using load and displacement sensing indentation experiments, J. Mater. Res. 7 (1992) 1564-1583

[10] A.C. Fischer-Cripps, Nanoindentation, Berlin: SpringerVerlag, New York, NY, 2002

[11] J.P. Ponthot, Unified stress update algorithms for the numerical simulation of large deformation elasto-plastic and elasto-viscoplastic processes, Int. J. Plastic. 18 (2002) 91-126

[12] P.Z. Berke, Numerical modeling of the surface and the bulk deformation in a small scale contact, Application to the nanoindentation interpretation and to the micromanipulation, $\mathrm{PhD}$ thesis, Université Libre de Bruxelles, Bruxelles, Belgium, 2008

[13] G. Rauchs, Optimization-based material parameter identification in indentation testing for finite strain elastoplasticity, ZAMM, J. Appl. Math. Mech. 86 (2006) 539562

[14] M. Dao, N. Chollacoop, K.J. Van Vliet, T.A. Venkatesh, S. Suresh, Computational modeling of the forward and reverse problems in instrumented sharp indentation, Acta Materialia 49 (2001) 3899-3918

[15] G. Bolzon, G. Maier, M. Panico, Material model calibration by indentation, imprint mapping and inverse analysis, Int. J. Solids Struct. 41 (2004) 2957-2975

[16] X. Chen, N. Ogasawara, M. Zhao, N. Chiba, On the uniqueness of measuring elastoplastic properties from indentation: the indistinguishable mystical materials, J. Mech. Phys. Sol. 55 (2007) 1618-1660

[17] S. Stupkiewicz, J. Korelc, M. Dutko, T. Rodic, Shape sensitivity analysis of large deformation frictional contact problems, Comput. Methods in Appl. Mech. Eng. 191 (2002) 3555-3581

[18] M. Bocciarelli, G. Bolzon, G. Maier, Parameter identification in anisotropic elastoplasticity by indentation and imprint mapping, Mech. Mat. 37 (2005) 855-868

[19] M. Mata, J. Alcala, The role of friction on sharp indentation, J. Mech. Phys. Sol. 52 (2004) 145-165

[20] A. Gouldstone, N. Chollacoop, M. Dao, J. Li, A.M. Minor, Y.L. Shen, Indentation across size scales and disciplines: Recent developments in experimentation and modeling, Acta Materialia 55 (2007) 4015-4039

[21] H.J. Albrecht, T. Hannach, A. Hase, A. Juritza, K. Muller, W.H. Muller, Nanoindentation: a suitable tool to determine local mechanical properties in microelectronic packages and materials?, Archive of Applied Mechanics 74 (2005) 728-738
[22] M. Vandamme, F.J. Ulm, Viscoelastic solutions for conical indentation, Int. J. Sol. Struct. 43 (2006) 3142-3165

[23] A.E. Giannakopoulos, Elastic and viscoelastic indentation of flat surfaces by pyramid indentors, J. Mech. Phys. Sol. 54 (2006) 1305-1332

[24] Y. Wang, D. Raabe, C. Kluber, F. Roters, Orientation dependence of nanoindentation pile-up patterns and of nanoindentation microtextures in copper single crystals, Acta Materialia 52 (2004) 2229-2238

[25] L. Cheng, X. Xia, L.E. Scriven, W.W. Gerberich, Spherical-tip indentation of viscoelastic material, Mech. Mat. 37 (2005) 213-226

[26] J.P. Ponthot, J.P. Kleinermann, A cascade optimization methodology for automatic parameter identification and shape/process optimization in metal forming simulation, Comput. Methods Appl. Mech. Eng. 195 (2006) 54725508

[27] Hysitron, TriboIndenter User's Manual, Hysitron Inc., 2005

[28] K.D. Bouzakis, N. Michailidis, S. Hadjiyiannis, G. Skordaris, G. Erkens, The effect of specimen roughness and indenter tip geometry on the determination accuracy of thin hard coatings stress-strain laws by nanoindentation, Materials Characterization 49 (2003) 149-156

[29] J.P. Ponthot, finite-element code. METAFOR, http:// metafor.ltas.ulg.ac.be/dokuwiki/, 2010

[30] J.L. Bucaille, E. Felder, G. Hochstetter, Identification of the viscoplastic behavior of a polycarbonate based on experiments and numerical modeling of the nanoindentation test, J. Mater. Sci. 37 (2002) 3999-4011

[31] J.M. Antunes, L.F. Menezes, J.V. Fernandes, Influence of Vickers tip imperfection on depth sensing indentation tests, Int. J. Sol. Struct. 44 (2007) 2732-2747

[32] S.M. Jeong, H.L. Lee, finite-element analysis of the tip deformation effect on nanoindentation hardness, Thin Solid Films 492 (2005) 173-179

[33] N. Yu, A.A. Polycarpou, T.F. Conry, Tip-radius effect in finite-element modeling of sub-50 nm shallow nanoindentation, Thin Solid Films 450 (2004) 295-303

[34] A.J.A. Winnubst, K. Keizer, A.J. Burggraaf, Mechanical properties and fracture behaviour of $\mathrm{ZrO}_{2}-\mathrm{Y}_{2} \mathrm{O}_{3}$ ceramics, J. Mater. Sci. 18 (1983) 1958-1966

[35] Z.M. Zhao, L. Zhang, Y.G. Song, W.G. Wang, H.B. Liu, Composition, microstructures and properties of $\mathrm{Al}_{2} \mathrm{O}_{3} / \mathrm{ZrO}_{2} \quad\left(\mathrm{Y}_{2} \mathrm{O}_{3}\right)$ self-growing ceramic composites prepared by combustion synthesis under high gravity, J. Phys.: Conf. Ser. 152 (2009) 012085

[36] X. Chen, J.J. Vlassak, Numerical study on the measurement of thin film mechanical properties by means of nanoindentation, J. Mater. Res. 16 (2001) 2974-2982 